\title{
Surgical wound management made easier and more cost-effective
}

\author{
ICHIRO AKAGI, KIYONORI FURUKAWA, MASAO MIYASHITA, \\ TERUO KIYAMA, AKIHISA MATSUDA, TSUTOMU NOMURA, HIROSHI MAKINO, \\ NOBUTOSHI HAGIWARA, KEN TAKAHASHI and EIJI UCHIDA \\ Division of Surgery for Organ Function and Biological Regulation, Nippon Medical School, Tokyo, Japan
}

Received July 5, 2011; Accepted November 11, 2011

DOI: $10.3892 / \mathrm{ol} .2012 .687$

\begin{abstract}
Evidence-based guidelines for the prevention of surgical site infection (SSI) have been published by the U.S. Centers for Disease Control and Prevention (CDC). According to these guidelines, a wound should usually be covered with a sterile dressing for 24 to $48 \mathrm{~h}$ when a surgical incision is closed primarily. However, it is not recommended that an incision be covered by a dressing beyond $48 \mathrm{~h}$. In this study, patients were stratified into two groups for analysis: patients whose surgical wound was sterilized and whose gauze was changed once daily until postoperative day 7 (7POD; group A); and patients whose surgical wound was sterilized and whose gauze was changed once daily until 2POD (group B). We evaluated the incidence of SSI, nursing hours and cost implications. The results showed that there was no significant difference in SSI occurrence between the two groups (group A, 10\% vs. group B, 7.3\%). By contrast, the average nursing time differed by $2.8 \mathrm{~min}$ (group A, 3.8 min vs. group B, 0.9 min). The material costs per patient were also reduced by $\$ 14.70$ (group A, $\$ 61.80$ vs group B, \$47.10). In conclusion, we applied our knowledge of the evidence-based CDC guidelines to determine whether 48-h wound management can be made easier, more uniform and more cost-effective compared to conventional wound management. The results of the present study showed that surgical wound management methods can be more convenient and inexpensive.
\end{abstract}

\section{Introduction}

Surgical wounds are given local care when healing; for example, by maintaining a moist wound environment to foster healing (1). There are numerous types of dressings (including gauze and bandages) and topical agents (including antiseptics

Correspondence to: Dr Ichiro Akagi, Division of Surgery for Organ Function and Biological Regulation, Nippon Medical School, 1-1-5 Sendagi, Tokyo 113-8602, Japan

E-mail: ichiro@nms.ac.jp

Key words: surgical wound management, surgical site infection and enzymes) available for surgical wound management. The ideal dressing for healing wounds has a number of features, including the ability to absorb and contain exudate without leakage, impermeability to water and bacteria, lack of particulate contaminants that may be left in the wound, and avoidance of wound trauma on dressing removal. However, dressings and topical agents are occasionally used inappropriately and uneconomically.

The scientific literature regarding the care of surgical wounds describes numerous advanced wound care modalities developed over the past 40 years. Among them, evidence-based guidelines for the prevention of surgical site infection (SSI) have been published by the U.S. Centers for Disease Control and Prevention (CDC) (2). This organization provides recommendations concerning the reduction of SSI risk. Each recommendation is classified on the basis of existing scientific data, theoretical rationale and applicability. According to these guidelines, the type of postoperative incision care is determined by whether the incision is closed primarily (i.e., the skin edges are re-approximated at the end of the operation), left open to be closed later, or left open to heal by second intention (2). When a surgical incision is closed primarily, as is usually the case, the incision is usually covered with a sterile dressing for 24 to $48 \mathrm{~h}$. Beyond $48 \mathrm{~h}$, it is unclear whether an incision should be covered by a dressing, or whether showering or bathing is detrimental to healing (2).

Most surgically sutured wounds are acute wounds that heal without complication in an expected time frame. When the incision is closed primarily, the wounds proceed through a specific cell and biochemical sequence of healing that comprises overlapping stages of hemostasis, inflammation, granulation and epithelialization within 48 h (2). Previously in our department, surgical wounds were sterilized and gauze was changed once daily until postoperative day 7 (7POD). This form of management for surgical wounds was inconsistent with the CDC guidelines, which raises the question of whether it is necessary to sterilize surgical wounds and change gauze once daily until 7POD.

In this study, patients were stratified into two groups for analysis: patients whose surgical wound was sterilized and whose gauze was changed once daily until 7POD; and patients whose surgical wound was sterilized and whose gauze was changed once daily until 2POD. We evaluated the incidence of SSI, nursing hours and cost implications. 
Table I. Patient characteristics.

\begin{tabular}{lcc}
\hline & $\begin{array}{c}\text { Group A } \\
(\mathrm{n}=40)\end{array}$ & $\begin{array}{c}\text { Group B } \\
(\mathrm{n}=41)\end{array}$ \\
\hline Male/female & $26 / 14$ & $28 / 13$ \\
Age (yrs.), mean \pm SD & $61.3 \pm 20.1$ & $63.2 \pm 14.9$ \\
Opened gastrointestinal tract & & \\
Gastric cancer & 8 & 10 \\
Colon cancer & 5 & 7 \\
Cholangiocarcinoma & 1 & 1 \\
Hepatocellular carcionoma & 1 & 3 \\
Pancreatic cancer & 1 & 3 \\
Appendicitis & 4 & 3 \\
Gallstone & 4 & 4 \\
Closed gastrointestinal tract & & \\
Breast cancer & 4 & 3 \\
Inguinal hernia & 12 & 7 \\
\hline
\end{tabular}

\section{Patients and methods}

Patients. A prospective study of all patients who underwent operative treatment between May 1 and October 31, 2003, was performed. These patients were selected from all patients who were treated during that time period at the Nippon Medical School Main Hospital, Japan. Study patients were all treated by surgeons in the surgery department. This was an institutional review board-approved study, with patient consent obtained for all study patients. Study inclusion criteria included diagnosis of gastroenterological disease, breast cancer, inguinal hernia, clinical stability, and adequate postoperative follow-up documentation. Exclusion criteria included coexisting diseases, steroid use, complications (including diabetes mellitus, hypertension, hyperlipidemia and pneumonia), aged $<15$ years and failure to return for postoperative follow-up. Data were collected on age, gender, surgical procedure, hospital charges and follow-up. Patients were stratified into two groups for analysis: group A, patients whose surgical wound was sterilized and whose gauze was changed once daily until 7POD; and group B, patients whose surgical wound was sterilized and whose gauze was changed once daily until 2POD.

The surgical wounds of group B were not managed but were carefully observed beyond $48 \mathrm{~h}$. The gauze surrounding the drainage tube was changed properly until the drainage tube was removed. Patient characteristics are shown in Table I. The two groups of patients were similar in terms of age, gender and diseases. The material cost per operation and nursing hours for changing the gauze were compared between the two groups. The prices of consumables, such as gauze, $10 \%$ isoGln liquid $\mathrm{TM}^{\circledR}, 0.2 \%$ Welpas $^{\circledR}$ and sterilized gloves, were obtained from the national medical aids reimbursement price list. The sterilized gauze was changed an average of once daily in this study. If a wound infection was suspected based on the appearance or odor of the wound or systemic signs (such as fever or tachycardia), the gauze dressing was removed and the

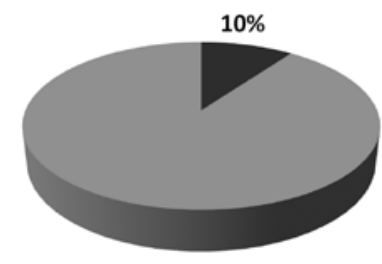

Group A

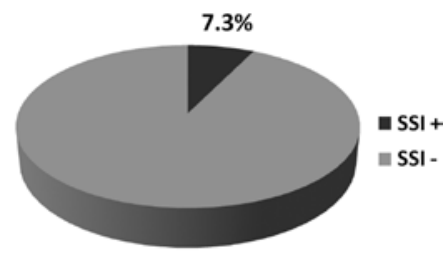

Group B
Figure 1. The rates of surgical site infection (SSI). There was no significant difference in SSI occurrence (group A, 10\%; group B, 7.3\%).

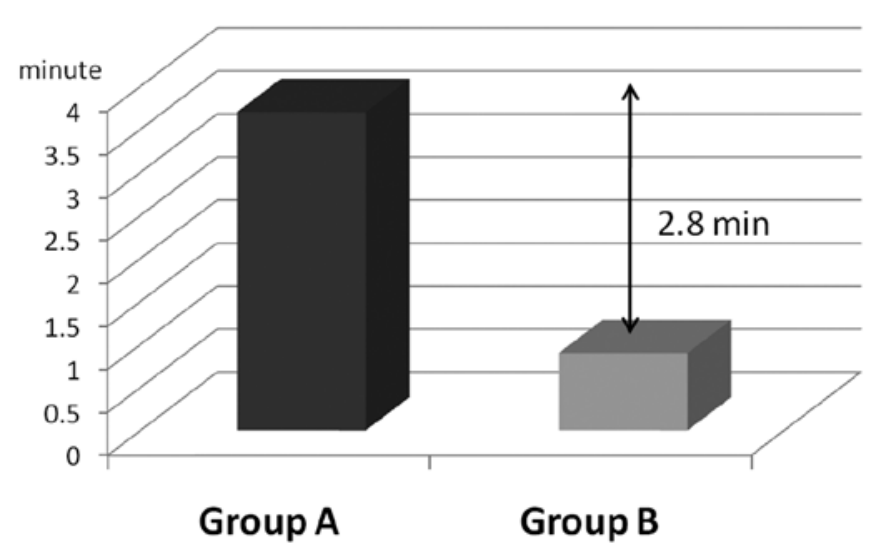

Figure 2. The average nursing time for changing the gauze differed by $2.8 \mathrm{~min}$ (71.1\%)-3.7 min for group A patients compared with 0.9 min for group B patients.

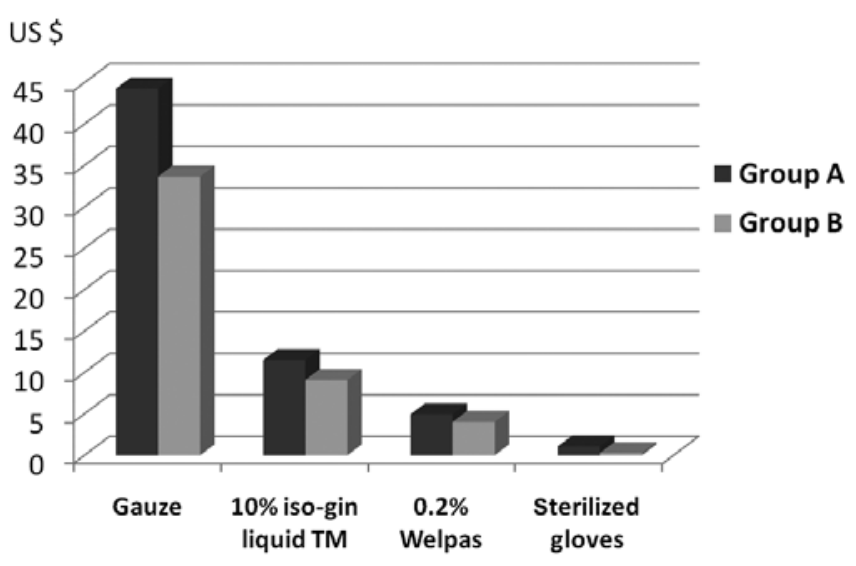

Figure 3. Mean material costs of surgical wound management per operation.

wound was inspected. Using a sterile technique, the wound was lavaged using saline.

\section{Results}

Rates of surgical site infection. In group A, there were 4 wound infections (10\%), all of which occurred in the midline wound with erythema and induration (Fig. 1). In group B, there were 3 wound infections $(7.3 \%)$, all of which occurred in the midline wound (Fig. 1). These wounds were reopened and 
lavaged. There was no significant difference in SSI occurrence between the two groups.

Clinical outcomes and treatment costs. It was anticipated that the implementation of evidence-based guidelines for wound management would lead to improved clinical outcomes. The average nursing time was reduced by $2.8 \mathrm{~min}(71.1 \%)$ $3.7 \mathrm{~min}$ for group A patients compared with $0.9 \mathrm{~min}$ for group B patients (Fig. 2). Wound management for 48 -h was also expected to reduce material costs. The material costs per patient were $\$ 61.80$ (gauze, $\$ 44.3 ; 10 \%$ isoGln liquid TM, \$11.50; 0.2\% Welpas, \$4.90; sterilized gloves, $\$ 1.10$ ) in group A and $\$ 47.10$ (gauze, $\$ 33.70 ; 10 \%$ isoGln liquid TM, \$9.10; 0.2\% Welpas, \$4.00; sterilized gloves, \$0.30) in group B (Fig. 3). The difference in material costs was $\$ 14.70$ (a $23.8 \%$ reduction).

\section{Discussion}

The increasing healthcare expenditure covered by the National Medical Insurance system has been a critical problem in Japan. Thus, the costs and consequences of surgical wound management should be considered a significant healthcare priority due to its prevalence and the amount of associated healthcare resource use. Surgical wounds are managed by clinicians, nurses and caregivers with essential systems of support, including specialized clinical training, links to allied health care professionals, established best practice guidelines, measuring and monitoring tools and focused commitment from health care organizations. The CDC guidelines do not recommend wound management using gauze-based dressings beyond $48 \mathrm{~h}$. The development of such guidelines is occasionally complicated by the heterogeneous nature of SSIs, which makes it difficult to generalize findings from a study of a specific patient population to a wider setting, and by the fact that the impact of various routine practices (such as wearing surgical gloves) cannot be evaluated for ethical or logistical reasons (2). This is the reason for the recent development in wound management not being well-recognized among patients and health care institutions at present.

To the best of our knowledge, this is the first prospective study comparing two principles of surgical wound management, i.e., conventional 7-day vs. 48-h wound management, in surgical patients with primary wound closure. Patients were only recruited from the surgical wards of the hospital for a number of reasons. First, selecting surgical patients limited patient and wound heterogeneity. Second, the nursing staff of these wards were most familiar with the two regimens of surgical wound care. The evidence from our study shows that, in this clinical setting, 48-h wound management does not lead to greater SSI or patient discomfort than conventional 7-day wound management and is likely to be more cost-effective. In closed surgical wounds, the main function of the gauze-based dressing is to absorb blood or hemoserous fluid in the immediate postoperative phase. By contrast, gauze-based dressings potentially cause avoidable pain and trauma on removal and typically harden in the wound, requiring softening by bathing prior to extraction (3). Such pain may impact on the health-related quality of life of the patient involved. Early removal of the gauze causes less pain and improved comfort on removal. It also allows surgical patients to take a shower or bath since the material had served as a protection of the wound from environmental influences (4). Moreover, it enables not only the clinician but also nurses to assess wound healing, the condition of the surrounding skin, exudate handling and the presence of infection at any time. These factors also contribute to the quality of life of the patient.

Forty-eight hour wound management requires fewer nursing hours for the changing of the gauze, which may lead to nursing staff being re-assigned to more appropriate duties. In addition, it contributes to a reduction in the overall cost of wound management. However, our results showed that a wound can be healed at a cost of $\$ 47.10$, assuming that the healing rate is constant throughout the process. This amount is low compared with the estimated cost using conventional management, which is at $\$ 61.80$. Although there are certain differences in health insurance systems among countries, this low cost of treating primary closed surgical wounds is likely to be of considerable interest to medical insurers and hospitals alike.

To promote the prevalence of evidence-based guidelines, certain institutes have an identified physician champion and coordinator. Theoretically, these roles differ in the way they may exert influence to achieve desired changes, but function as change agents, using interpersonal contact and strong communication skills to build trust, promote the use of new practices, and thus affect behavior (5). The ability of opinion leaders to change practices is known to be somewhat limited, in part because studies evaluating this intervention have not described consistent methods for identifying or engaging opinion leaders (6). The cooperative structure between clinicians and nurses has resulted in the adoption of all recommended evidence-based guidelines.

There are a number of limitations to our study. One involved the difficulty in analyzing the use of antibiotics, since antibiotics were used not only for serious underlying intra-abdominal infections, where the duration and type of antibiotics in part depended on the clinical response in each patient, but also for a variety of concomitant indications (such as pneumonia or line sepsis). Therefore, it was not ethical to restrict the use of antibiotics in accordance with a specified protocol. Second, it is more likely that other factors played a more significant role in determining the hospital stay than surgical wound management. This is the reason for the length of stay not being taken into consideration in this study. Third, the investigators evaluating the wounds could not be masked, and this may have introduced a potential bias depending on each investigator's preference for skin closure. Future studies may benefit from the use of independent assessors who are blinded to study treatment allocation.

In conclusion, we applied previous knowledge of the evidence-based CDC guidelines to determine whether 48-h wound management can be made easier, more uniform, and more cost-effective compared with conventional wound management. The results of the present study showed that surgical wound management methods can be more convenient and inexpensive. Guidelines also change over time as new methods are adopted. Adaptation and re-evaluation may therefore be required when new evidence regarding surgical wound management is published. 


\section{Acknowledgements}

The authors thank the hospital committees for endorsing this study.

\section{References}

1. Schultz GS, Sibbald RG, Falanga V, et al: Wound bed preparation: a systematic approach to wound management. Wound Repair Regen 11 (Suppl 1): 1-28, 2003.

2. Mangram AJ, Horan TC, Pearson ML, Silver LC and Jarvis WR: Guideline for prevention of surgical site infection, 1999. Centers for Disease Control and Prevention (CDC) Hospital Infection Control Practices Advisory Committee. Am J Infect Control 27: 97-132; Quiz 133-134; Discussion 196, 1999.
3. Hollinworth $\mathrm{H}$ and Collier M: Nurses' views about pain and trauma at dressing changes: results of a national survey. J Wound Care 9: 369-373, 2000.

4. Hermans MH: Clinical benefit of a hydrocolloid dressing in closed surgical wounds. J ET Nurs 20: 68-72, 1993.

5. Thompson GN, Estabrooks CA and Degner LF: Clarifying the concepts in knowledge transfer: a literature review. J Adv Nurs 53: 691-701, 2006.

6. Lansisalmi H, Kivimaki M, Aalto P and Ruoranen R: Innovation in healthcare: a systematic review of recent research. Nurs Sci Q 19: 65-72, 2006. 